\title{
TORSION POINTS IN FAMILIES OF DRINFELD MODULES
}

\author{
D. GHIOCA AND L.-C. HSIA
}

\begin{abstract}
Let $\Phi^{\lambda}$ be an algebraic family of Drinfeld modules defined over a field $K$ of characteristic $p$, and let $\mathbf{a}, \mathbf{b} \in K[\lambda]$. Assume that neither $\mathbf{a}(\lambda)$ nor $\mathbf{b}(\lambda)$ is a torsion point for $\Phi^{\lambda}$ for all $\lambda$. If there exist infinitely many $\lambda \in \bar{K}$ such that both $\mathbf{a}(\lambda)$ and $\mathbf{b}(\lambda)$ are torsion points for $\Phi^{\lambda}$, then we show that for each $\lambda \in \bar{K}$, we have that $\mathbf{a}(\lambda)$ is torsion for $\Phi^{\lambda}$ if and only if $\mathbf{b}(\lambda)$ is torsion for $\Phi^{\lambda}$. In the case $\mathbf{a}, \mathbf{b} \in K$, then we prove in addition that $\mathbf{a}$ and $\mathbf{b}$ must be $\overline{\mathbb{F}_{p}}$-linearly dependent.
\end{abstract}

\section{INTRODUCTION}

Lang 22 proved that if a curve $C \subset \mathbb{A}^{2}$ contains infinitely many points whose coordinates are both roots of unity, then $C$ is the zero set of an equation of the form $X^{m} Y^{n}=\alpha$, for some $m, n \in \mathbb{Z}$ (not both equal to 0 ) and some root of unity $\alpha$. In this case we note that if $C$ projects dominantly on both axis, then for each point $(x, y) \in C$ we know that $x$ is a root of unity if and only if $y$ is a root of unity. In particular, the following result is a corollary of Lang's Theorem.

Corollary 1.1. Let $F_{1}, F_{2} \in \mathbb{C}(z)$ be nonconstant rational maps such that there exist infinitely many $\lambda \in \mathbb{C}$ such that both $F_{1}(\lambda)$ and $F_{2}(\lambda)$ are roots of unity. Then $F_{1}$ and $F_{2}$ are multiplicatively dependent, and therefore for each $\lambda \in \mathbb{C}, F_{1}(\lambda)$ is a root of unity if and only if $F_{2}(\lambda)$ is a root of unity.

Lang's result is a special case of the Manin-Mumford Conjecture (proven by Raynaud [28, 29] for abelian varieties, and by Hindry [20, for semiabelian varieties). The Manin-Mumford Conjecture (in its most general form asked by Lang) predicts that the set of torsion points of a semiabelian variety $G$ defined over $\mathbb{C}$ is not Zariski dense in a subvariety $V$ of $G$, unless $V$ is a translate of an algebraic subgroup of $G$ by a torsion point. Pink and Zilber have suggested extending the Manin-Mumford conjecture to a more general question regarding unlikely intersections between a subvariety $V$ of a semiabelian scheme $G$ and algebraic subgroups of the fibers of $G$ having codimension greater than the dimension of $V$ (see [5, 19, 24, 25, 26]).

Masser and Zannier [24, 25] study the Pink-Zilber Conjecture when $G$ is the square of the Legendre family of elliptic curves. We state below a special case of their theorem.

Theorem 1.2 (Masser-Zannier). For each $\lambda \in \mathbb{C} \backslash\{0,1\}$, let $E_{\lambda}$ be the elliptic curve given by the equation $Y^{2}=X(X-1)(X-\lambda)$. Let $P_{\lambda}$ and $Q_{\lambda}$ be two given

Date: June 16, 2018.

2010 Mathematics Subject Classification. Primary 37P05; Secondary 37P10.

Key words and phrases. Drinfeld modules; torsion points.

The first author was partially supported by an NSERC Discovery Grant. The second author was partially supported by the National Center of Theoretical Sciences of Taiwan and NSC Grant 99-2115-M-003-012-MY3. 
families of points on $E_{\lambda}$ depending algebraically on the parameter $\lambda$. Suppose there exist infinitely many $\lambda \in \mathbb{C}$ such that both $P_{\lambda}$ and $Q_{\lambda}$ are torsion points for $E_{\lambda}$. Then the points $P_{\lambda}$ and $Q_{\lambda}$ are linearly dependent over $\mathbb{Z}$ on the generic fiber of the elliptic surface $E_{\lambda}$.

Both Lang's result and Masser-Zannier's result are special cases of the following problem. Let $\left\{G_{\lambda}\right\}$ be an algebraic family of algebraic groups, and let $\left\{P_{\lambda}\right\}$ and $\left\{Q_{\lambda}\right\}$ be two algebraic families of points on $G_{\lambda}$. If there exist infinitely many $\lambda$ such that both $P_{\lambda}$ and $Q_{\lambda}$ are torsion points, then at least one of the following three properties holds:

(1) $P_{\lambda}$ is torsion for all $\lambda$.

(2) $Q_{\lambda}$ is torsion for all $\lambda$.

(3) for each $\lambda, P_{\lambda}$ is a torsion point if and only if $Q_{\lambda}$ is a torsion point.

In the arithmetic theory of function fields of positive characteristic, Drinfeld modules play a role similar to elliptic curves over number fields. Hence, it is natural to ask if there exist results for family of Drinfeld mdoules that are parallel to those obtained by Masser and Zannier. In this paper we study the first instance of the above problem in characteristic $p$, where $G_{\lambda}:=\left(\mathbb{G}_{a}, \Phi^{\lambda}\right)$ is the constant family of additive group schemes endowed with the action of a Drinfeld module $\Phi^{\lambda}$ (which belongs to an algebraic family of such Drinfeld modules). More precisely, let $K$ be a field extension of $\mathbb{F}_{q}(t)$ and let $K(z)$ be the rational function field in variable $z$. Let $\Phi: \mathbb{F}_{q}[t] \rightarrow \operatorname{End}_{K(z)}\left(\mathbb{G}_{a}\right)$ be a Drinfeld module defined over $K(z)$ (See Section 2 for details). Equivalently, $\Phi$ is also regarded as a family of Drinfeld modules by specialization. That is, by letting $z=\lambda$ for $\lambda \in \bar{K}$ we obtain an algebraic family of Drinfeld modules, denoted by $\Phi^{\lambda}$ which are defined over $\bar{K}$. Then we are able to prove that the above trichotomy must hold. The following result is a special case of our more general result (Theorem 2.4).

Theorem 1.3. Let $r \geq 2$ be an integer, let $q$ be a power of a prime number $p$, let $K$ be a field extension of $\mathbb{F}_{q}(t)$, and let $g_{1}, \ldots, g_{r-1} \in K[z]$. We let $\Phi: \mathbb{F}_{q}[t] \longrightarrow$ $\operatorname{End}_{K(z)}\left(\mathbb{G}_{a}\right)$ be the family of Drinfeld modules defined by

$$
\Phi_{t}(x)=t x+\sum_{i=1}^{r-1} g_{i}(z) x^{q^{i}}+x^{q^{r}} .
$$

Let $\mathbf{a}, \mathbf{b} \in K[z]$ and assume there exist infinitely many $\lambda \in \bar{K}$ such that both $\mathbf{a}(\lambda)$ and $\mathbf{b}(\lambda)$ are torsion points for $\Phi^{\lambda}$. Then at least one of the following properties holds:

(1) a is a torsion point for $\Phi$.

(2) $\mathbf{b}$ is a torsion point for $\Phi$.

(3) for each $\lambda \in \bar{K}, \mathbf{a}(\lambda)$ is a torsion point for $\Phi^{\lambda}$ if and only if $\mathbf{b}(\lambda)$ is a torsion point for $\Phi^{\lambda}$.

For certain families of Drinfeld modules and for constant starting points $\mathbf{a}, \mathbf{b}$ (i.e., no dependence on $z$ ) we can give an explicit condition of when there exist infinitely many $\lambda \in \bar{K}$ such that both $\mathbf{a}$ and $\mathbf{b}$ are torsion points for $\Phi^{\lambda}$.

Theorem 1.4. Let $r \geq 2$ be an integer and let $K$ be a field extension of $\mathbb{F}_{q}(t)$ such that $\mathbb{F}_{q}$ is algebraically closed in $K$. Let $\mathbf{a}, \mathbf{b} \in K$ and let $\Phi: \mathbb{F}_{q}[t] \longrightarrow \operatorname{End}_{K(z)}\left(\mathbb{G}_{a}\right)$ be the family of Drinfeld modules given by

$$
\Phi_{t}(x)=t x+z x^{q}+x^{q^{r}} .
$$


Then there exist infinitely many $\lambda \in \bar{K}$ such that both $\mathbf{a}$ and $\mathbf{b}$ are torsion points for $\Phi^{\lambda}$, if and only if $\mathbf{a}$ and $\mathbf{b}$ are linearly dependent over $\mathbb{F}_{q}$.

Going in the opposite direction, when $K$ is a constant field extension of $\mathbb{F}_{q}(t)$ we can give a more precise relation between $\mathbf{a}$ and $\mathbf{b}$.

Theorem 1.5. Let $r \geq 2$ be an integer, let $q$ be a power of a prime number $p$, let $\mathbf{a}, \mathbf{b} \in K=\overline{\mathbb{F}_{q}}(t)$ and let $\Phi: \mathbb{F}_{q}[t] \longrightarrow \operatorname{End}\left(\mathbb{G}_{a}\right)$ be the family of Drinfeld modules given by

$$
\Phi_{t}(x)=t x+z x^{q}+x^{q^{r}} .
$$

Then there exist infinitely many $\lambda \in \bar{K}$ such that both $\mathbf{a}$ and $\mathbf{b}$ are torsion points for $\Phi^{\lambda}$, if and only if $\mathbf{a}$ and $\mathbf{b}$ are linearly dependent over $\mathbb{F}_{q}$.

Both Theorems 1.4 and 1.5 should be true without any extra condition on the (constant) starting points a and $\mathbf{b}$. However, our methods cannot be extended to this general case. In order to prove Theorems 1.4 and 1.5 we employ a finer analysis of the valuation of torsion points for Drinfeld modules which fails for arbitrary extension fields $K$ of $\mathbb{F}_{q}(t)$.

A question analogous to the problem studied by Masser and Zannier in the setting of arithmetic dynamics is the following. Given complex numbers $a$ and $b$ and an integer $d \geq 2$, when do there exist infinitely many $\lambda \in \mathbb{C}$ such that both $a$ and $b$ are preperiodic for the action of $f_{\lambda}(x):=x^{d}+\lambda$ on $\mathbb{C}$ ? This question was first raised by Zannier at an American Institute of Mathematics workshop in 2008 and studied by Baker and DeMarco in [1. They show that this happens if and only if $a^{d}=b^{d}$. In [16], the authors together with Tucker extended Baker-DeMarco's result to general families of polynomials. On the other hand, a Drinfeld module can be viewed as a collection of (additive) polynomials acting on the affine line so we can apply the techniques and results that have been developed in arithmetic dynamics into the study of Drinfeld modules, especially to Diophantine problems arising from Drinfeld modules; this point of view is proven to be useful in our paper. The problem studied in this paper naturally can be viewed as an analogue of the problems from [1] and [16] in the setting of Drinfeld modules. Hence, our method for studing the question raised above follows closely the methods used in 1 . We will apply the techniques used in 16 to our situation. The reader will find materials in Section 3 and results in Section 4 parallel to [16, Sect. 4-6]. However, as we work in the world of positive characteristic, we can not expect that all the techniques developed in [1] and [16] can be applied to our situation. This paper reveals the differences and difficulties in the study of the same questions in the setting of Drinfeld modules. In particular, we cannot use complex analysis in order to derive the explicit relations between $\mathbf{a}$ and $\mathbf{b}$ as in Theorems 1.4 and 1.5- instead we use arguments from valuation theory which are amenable to Drinfeld modules.

The plan of our paper is as follows. In Section 2 we set up our notation and state our main result (Theorem 2.4), and then describe the method of our proof. In Section 3 we give a brief overview of Berkovich spaces. Then, in Section 4 we compute the capacities of the generalized $v$-adic Mandelbrot sets associated to a generic point $\mathbf{c}$ for a family of Drinfeld modules. We proceed with our proof of Theorem 2.4 in Section 5. Then, in Section 6 we conclude our paper by proving Theorems 1.3, 1.4 and 1.5.

Acknowledgments. The authors thank Thomas Tucker for several useful conversations. 


\section{Preliminary and Statement of the Main Results}

In this section, we give a brief review of the theory of Drinfeld modules and height functions that is relevant to our discussion below. Throughout the paper, we let $q$ be a power of a prime $p$ and let $\mathbb{F}_{q}$ be a finite field of $q$ elements.

2.1. Drinfeld Modules. We first define Drinfeld modules (of generic characteristic) - for more details, see [18]. Let $L$ be a field extension of the rational function field $\mathbb{F}_{q}(t)$. A Drinfeld $\mathbb{F}_{q}[t]$-module defined over $L$ is an $\mathbb{F}_{q}$-algebra homomorphism $\Phi: \mathbb{F}_{q}[t] \longrightarrow \operatorname{End}_{L}\left(\mathbb{G}_{a}\right)$ such that

$$
\Phi_{t}(x):=t x+\sum_{i=1}^{r-1} a_{i} x^{q^{i}}+x^{q^{r}}, \text { with } a_{i} \in L \quad \text { for all } i=1, \ldots, r-1
$$

where $\Phi_{a}$ denotes the image of $a \in \mathbb{F}_{q}[t]$ under $\Phi$ and $r \geq 1$ is called the rank of $\Phi$. Note that as an $\mathbb{F}_{q}$-algebra homomorphism, $\Phi$ is uniquely determined by the action of $\Phi_{t}$ on $\mathbb{G}_{a}$. In general, $\Phi_{t}(x)$ is not required to be monic in $x$. However, at the expense of replacing $\Phi$ by a Drinfeld module which is conjugated to it, i.e.

$$
\Psi_{t}(x)=\gamma^{-1} \Phi_{t}(\gamma x)
$$

for a suitable $\gamma \in \bar{L}$ we obtain that $\Psi_{t}$ is monic. Note that $\Psi$ is defined over $L(\gamma)$ which is a finite field extension of $L$.

A point $x \in \bar{L}$ is called torsion for $\Phi$ if there exists a nonzero $a \in \mathbb{F}_{q}[t]$ such that $x$ is in the kernel of $\Phi_{a}$. We denote by $\Phi_{\text {tor }}$ the set of all torsion points for $\Phi$. It is immediate to see that $x$ is torsion if and only if its orbit under the action of $\Phi_{t}$ is finite, i.e. $x$ is preperiodic for the map $\Phi_{t}$. Note that if $\Psi$ is a Drinfeld module conjugated to $\Phi$ as in (2.1), then $x \in \Phi_{\text {tor }}$ if and only if $\gamma^{-1} x \in \Psi_{\text {tor }}$.

2.2. Canonical heights. Let $K$ be a finitely generated transcendental extension of $\mathbb{F}_{q}$. At the expense of replacing $K$ by a finite extension and replacing $\mathbb{F}_{q}$ with its algebraic closure in this finite extension, we may assume there exists a smooth projective, geometrically irreducible variety $\mathcal{V}$ defined over $\mathbb{F}_{q}$ whose function field is $K$ (see [11]). We let then $\Omega_{K}$ be the set of places of $K$ corresponding to the codimension one irreducible subvarieties of $\mathcal{V}$. Then for each $v \in \Omega_{K}$ there exists a positive integer $N_{v}$ such that for all $\alpha \in K^{*}$ we have $\prod_{v \in \Omega}|\alpha|_{v}^{N_{v}}=1$ where for $v \in \Omega_{K}$, the corresponding absolute value is denoted by $|\cdot|_{v}$ (for more details see [23, $\S 2.3]$ or $[6, \S 1.4 .6])$. We note that if $x \in K$ is a $v$-adic unit for all $v \in \Omega_{K}$ then $x \in \mathbb{F}_{q}$.

Let $\mathbb{C}_{v}$ be a fixed completion of the algebraic closure of a completion $K_{v}$ of $\left(K,|\cdot|_{v}\right)$. Note that there is a unique extension of $|\cdot|_{v}$ to an absolute value on $\mathbb{C}_{v}$. By abuse of notation, we still denote this extension by $|\cdot|_{v}$. Let $\Phi$ be a Drinfeld module of rank $r$ defined over $\mathbb{C}_{v}$. Following Poonen [27] and Wang [30, for each $x \in \mathbb{C}_{v}$, the local canonical height of $x$ is defined as follows

$$
\widehat{\mathrm{h}}_{\Phi, v}(x):=\lim _{n \rightarrow \infty} \frac{\log ^{+}\left|\Phi_{t^{n}}(x)\right|_{v}}{q^{r n}},
$$

where by $\log ^{+} z$ we always denote $\log \max \{z, 1\}$ (for any real number $z$ ). It is immediate that $\widehat{\mathrm{h}}_{\Phi, v}\left(\Phi_{t^{i}}(x)\right)=q^{i r} \widehat{\mathrm{h}}_{\Phi, v}(x)$ and thus $\widehat{\mathrm{h}}_{\Phi, v}(x)=0$ whenever $x \in \Phi_{\text {tor }}$. 
Now, if $f(x)=\sum_{i=0}^{d} a_{i} x^{i}$ is any polynomial defined over $\mathbb{C}_{v}$, then $|f(x)|_{v}=$ $\left|a_{d} x^{d}\right|>|x|_{v}$ when $|x|_{v}>r_{v}$, where

$$
r_{v}=r_{v}(f):=\max \left\{1, \max \left\{\left|\frac{a_{i}}{a_{d}}\right|^{\frac{1}{d-i}}\right\}_{0 \leq i<d}\right\} .
$$

Moreover, for a Drinfeld module $\Phi$, if $|x|_{v}>r_{v}\left(\Phi_{t}\right)$ then $\widehat{\mathrm{h}}_{\Phi, v}(x)=\log |x|_{v}>0$. For more details see [17] and [21].

We fix an algebraic closure $\bar{K}$ of $K$, and for each $v \in \Omega_{K}$ we fix an embedding $\bar{K} \hookrightarrow \mathbb{C}_{v}$. The global canonical height $\widehat{\mathrm{h}}_{\Phi}(x)$ associated to the Drinfeld module $\Phi$ was first introduced by Denis [12] (Denis defined the global canonical heights for general $T$-modules which are higher dimensional analogue of Drinfeld modules). For each $x \in \bar{K}$, the global canonical height is defined as

$$
\widehat{\mathrm{h}}_{\Phi}(x)=\lim _{n \rightarrow \infty} \frac{h\left(\Phi_{t^{n}}(x)\right)}{q^{r n}},
$$

where $h$ is the usual (logarithmic) Weil height on $\bar{K}$. As shown in 27] and [30, the global canonical height decomposes into a sum of the corresponding local canonical heights. Furthermore, $\widehat{\mathrm{h}}_{\Phi}(x)=0$ if and only if $x \in \Phi_{\text {tor }}$ (see [30, Proposition 3 (v)]).

Remark 2.3. (1). We note that the theory of canonical height associated to a Drinfeld module is a special case of the canonical heights associated to morphisms on algebraic varieties developed by Call and Silverman (see [8] for details).

(2) The definition for the canonical height functions given above seems to depend on the particular choice of the map $\Phi_{t}$. On the other hand, one can define the canonical heights $\widehat{\mathrm{h}}_{\Phi}$ as in [12] by letting

$$
\widehat{\mathrm{h}}_{\Phi}(x)=\lim _{\operatorname{deg}(R) \rightarrow \infty} \frac{h\left(\Phi_{R}(x)\right)}{q^{r \operatorname{deg}(R)}},
$$

and similar formula for canonical local heights $\widehat{\mathrm{h}}_{\Phi, v}(x)$ where $R$ runs through all non-constant polynomials in $\mathbb{F}_{q}[t]$. In [27] and [30] it is proven that both definitions yield the same height function.

Let $\Phi: \mathbb{F}_{q}[t] \rightarrow \operatorname{End}_{L}\left(\mathbb{G}_{a}\right)$ be the Drinfeld module as in Theorem 1.3 with $L=K(z)$. Then, our main result is the following.

Theorem 2.4. Let let $K$ be a field extension of $\mathbb{F}_{q}(t), r \geq 2$ be an integer, and let $g_{1}, \ldots, g_{r-1} \in K[z]$. We let $\Phi: \mathbb{F}_{q}[t] \longrightarrow \operatorname{End}_{K(z)}\left(\mathbb{G}_{a}\right)$ be the family of Drinfeld modules defined by

$$
\Phi_{t}(x)=t x+\sum_{i=1}^{r-1} g_{i}(z) x^{q^{i}}+x^{q^{r}} .
$$

Let $\mathbf{a}, \mathbf{b} \in K[z]$ and assume the following inequality holds:

$$
\min \{\operatorname{deg}(\mathbf{a}), \operatorname{deg}(\mathbf{b})\}>\max \left\{\frac{\operatorname{deg}\left(g_{1}\right)}{q^{r}-q}, \ldots, \frac{\operatorname{deg}\left(g_{r-1}\right)}{q^{r}-q^{r-1}}\right\} .
$$

If there exist infinitely many $\lambda \in \bar{K}$ such that both $\mathbf{a}(\lambda)$ and $\mathbf{b}(\lambda)$ are torsion points for $\Phi^{\lambda}$, then for each $\lambda \in \bar{K}$ we have that $\mathbf{a}(\lambda)$ is torsion for $\Phi^{\lambda}$ if and only if 
$\mathbf{b}(\lambda)$ is torsion for $\Phi^{\lambda}$. Furthermore, in this case, if $C_{\mathbf{a}}$ and $C_{\mathbf{b}}$ are the leading coefficients of $\mathbf{a}$, respectively of $\mathbf{b}$, then $\frac{C_{\mathbf{a}}^{\operatorname{deg}(\mathbf{b})}}{C_{\mathbf{b}}^{\operatorname{deg}(\mathbf{a})}} \in \overline{\mathbb{F}_{p}}$.

Note that inequality (2.5) prevents $\mathbf{a}$ and $\mathbf{b}$ to be torsion points for all $\lambda$ (see Proposition 4.2).

Remark 2.6. One can consider a more general Drinfeld module such that the additive group $\mathbb{G}_{a}$ is equipped with an action by a ring $A$ cosisting of all regular functions on a smooth projective curve defined over $\mathbb{F}_{q}$ with a fixed point removed. In our definition above, the curve is $\mathbb{P}^{1}$ with the distinguished point at infinity. However, we lose no generality by restricting ourselves to the case $A=\mathbb{F}_{q}[t]$ since always $\mathbb{F}_{q}[t]$ embeds into such a ring $A$, and moreover, the notion of torsion is identical for $\Phi$ and for its restriction to $\mathbb{F}_{q}[t]$.

Our results and proofs are inspired by the results of [1] so that the strategy for the proof of Theorem 2.4 essentially follows the ideas in the paper 11. However there are different technical details in our proofs. Also, Drinfeld modules are better vehicle for the method from [16] which generalizes [1].

Following [1, the strategy to prove Theorem 2.4] is the following. For the family of Drinfeld modules $\Phi$, We define the $v$-adic generalized Mandelbrot sets $M_{\mathbf{c}, v}$ (inside the Berkovich affine line) associated to any $\mathbf{c} \in K[z]$ similar to that introduced in 11. We then use the equidistribution result discovered independently by BakerRumely [2, Chambert-Loir [10] and Favre-Rivera-Letelier [13, 14] to deduce that the corresponding $v$-adic Mandelbrot sets $M_{\mathbf{a}, v}$ and $M_{\mathbf{b}, v}$ for the given points a and $\mathbf{b}$ are equal for each $v \in \Omega_{K}$. Here we use the version obtained by Baker and Rumely [2] to connect the equidistribution result to arithmetic capacities which is used in the final step of the proof to show that the leading coeffients of $\mathbf{a}$ and $\mathbf{b}$ have the desired relation.

In order to apply the equidistribution results and capacity theory over nonarchimedean fields mentioned above, we need to introduce the Berkovich space associated to the affine line which we give an overview in the next section.

\section{Equidistribution Theorem and Berkovich spaces}

As mentioned above, we will need to apply the arithmetic equidistribution discovered independently by Baker-Rumely, Chambert-Loir and Favre-Rivera-Letelier. When the base field is a nonarchimedean field, the equidistribution theorem is best stated over the Berkovich space associated to the underlying variety in question. For the convenience of the reader, we give a review on the Berkovich spaces in this section.

We use the version of equidistribtution theorem obtained by Baker and Rumely which connects the equidistribution result to the theory of arithmetic capacities. Hence, the material presented in this section is mainly from the book 3 by Baker and Rumely. The main body of this Section is taken from [16, Section 4] which is written according to the summary in [1. For more details on the arithmetic equidistribution as well as a detailed introduction to Berkovich line we refer the reader to the book [3].

Let $K$ be a field of characteristic $p$ endowed with a product formula, and let $\Omega_{K}$ be the set of its inequivalent absolute values. For each $v \in \Omega_{K}$, we let $\mathbb{C}_{v}$ be the completion of an algebraic closure of the completion of $K$ at $v$. Let $\mathbb{A}_{\text {Berk, }}^{1} \mathbb{C}_{v}$ 
denote the Berkovich affine line over $\mathbb{C}_{v}$ (see [4] or [3, $\left.\S 2.1\right]$ for details). Then $\mathbb{A}_{\text {Berk, }, \mathbb{C}_{v}}^{1}$ is a locally compact, Hausdorff, path-connected space containing $\mathbb{C}_{v}$ as a dense subspace (with the topology induced from the $v$-adic absolute value). As a topological space, $\mathbb{A}_{\text {Berk, } \mathbb{C}_{v}}^{1}$ is the set consisting of all multiplicative seminorms, denoted by $[\cdot]_{x}$, on $\mathbb{C}_{v}[T]$ extending the absolute value $|\cdot|_{v}$ on $\mathbb{C}_{v}$ endowed with the weakest topology such that the map $z \mapsto[f]_{z}$ is continuous for all $f \in \mathbb{C}_{v}[T]$. The set of seminorms can be described as follows. If $\left\{D\left(a_{i}, r_{i}\right)\right\}_{i}$ is any decreasing nested sequence of closed disks $D\left(c_{i}, r_{i}\right)$ centered at points $c_{i} \in \mathbb{C}_{v}$ of radius $r_{i} \geq 0$, then the map $f \mapsto \lim _{i \rightarrow \infty}[f]_{D\left(c_{i}, r_{i}\right)}$ defines a multiplicative seminorm on $\mathbb{C}_{v}[T]$ where $[f]_{D\left(c_{i}, r_{i}\right)}$ is the sup-norm of $f$ over the closed disk $D\left(a_{i}, r_{i}\right)$. Berkovich's classification theorem says that there are exactly four types of points, Type I, II, III and IV. The first three types of points can be described in terms of closed disks $\zeta=D(c, r)=\cap D\left(c_{i}, r_{i}\right)$ where $c \in \mathbb{C}_{v}$ and $r \geq 0$. The corresponding multiplicative seminorm is just $f \mapsto[f]_{D(c, r)}$ for $f \in \mathbb{C}_{v}[T]$. Then, $\zeta$ is of Type I, II or III if and only if $r=0, r \in\left|\mathbb{C}_{v}^{*}\right|_{v}$ or $r \notin\left|\mathbb{C}_{v}^{*}\right|_{v}$ respectively. As for Type IV points, they correspond to sequences of decreasing nested disks $D\left(c_{i}, r_{i}\right)$ such that $\cap D\left(c_{i}, r_{i}\right)=\emptyset$ and the multiplicative seminorm is $f \mapsto \lim _{i \rightarrow \infty}[f]_{D\left(c_{i}, r_{i}\right)}$ as described above. For details, see [4] or [3]. For $\zeta \in \mathbb{A}_{\text {Berk, } \mathbb{C}_{v}}^{1}$, we sometimes write $|\zeta|_{v}$ instead of $[T]_{\zeta}$.

In order to apply the main equidistribution result from [3, Theorem 7.52], we recall the potential theory on the affine line over $\mathbb{C}_{v}$. The right setting for nonarchimedean potential theory is the potential theory on $\mathbb{A}_{\text {Berk, } \mathbb{C}_{v}}^{1}$ developed in $[3]$. We quote part of a nice summary of the theory from [1, $\S 2.2$ and 2.3] without going into details. We refer the reader to 1, 3, for all the details and proofs. Let $E$ be a compact subset of $\mathbb{A}_{\text {Berk, }, \mathbb{C}_{v}}^{1}$. Then analogous to the complex case, the logarithmic capacity $\gamma(E)=e^{-V(E)}$ and the Green's function $G_{E}$ of $E$ relative to $\infty$ can be defined where $V(E)$ is the infimum of the energy integral with respect to all possible probability measures supported on $E$. More precisely,

$$
V(E)=\inf _{\mu} \iint_{E \times E}-\log \delta(x, y) d \mu(x) d \mu(y),
$$

where the infimum is computed with respect to all probability measures $\mu$ supported on $E$, while for $x, y \in \mathbb{A}_{\text {Berk, } \mathbb{C}_{v}}^{1}$, the function $\delta(x, y)$ is the Hsia kernel (see [3, Proposition 4.1]):

$$
\delta(x, y):=\limsup _{\substack{z, w \in \mathbb{C}_{v} \\ z \rightarrow x, w \rightarrow y}}|z-w|_{v} .
$$

The following are basic properties of the logarithmic capacity of $E$.

- If $E_{1}, E_{2}$ are two compact subsets of $\mathbb{A}_{\text {Berk, } \mathbb{C}_{v}}^{1}$ such that $E_{1} \subset E_{2}$ then $\gamma\left(E_{1}\right) \leq \gamma\left(E_{2}\right)$.

- If $E=\{\zeta\}$ where $\zeta$ is a Type II or III point corresponding to a closed disk $D(c, r)$ then $\gamma(E)=r>0$. [3, Example 6.3]. (This can be viewed as analogue of the fact that a closed disk $D(c, r)$ of positive radius $r$ in $\mathbb{C}_{v}$ has logarithmic capacity $\gamma(D(c, r))=r$.)

If $\gamma(E)>0$, then the exists a unique probability measure $\mu_{E}$ attaining the infimum of the energy integral. Furthermore, the support of $\mu_{E}$ is contained in the boundary

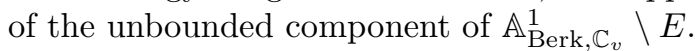

The Green's function $G_{E}(z)$ of $E$ relative to infinity is a well-defined nonnegative real-valued subharmonic function on $\mathbb{A}_{\text {Berk, } \mathbb{C}_{v}}^{1}$ which is harmonic on $\mathbb{A}_{\text {Berk, }, \mathbb{C}_{v}}^{1} \backslash E$ 
(in the sense of [3, Chapter 8]). If $\gamma(E)=0$, then there exists no Green's function associated to the set $E$. Indeed, as shown in [3, Proposition 7.17, page 151], if $\gamma(\partial E)=0$ then there exists no nonconstant harmonic function on $\mathbb{A}_{\text {Berk, }, \mathbb{C}_{v}}^{1} \backslash E$ which is bounded below (this is the Strong Maximum Principle for harmonic functions defined on Berkovich spaces). The following result is [1, Lemma 2.2 and 2.5], and it gives a characterization of the Green's function of the set $E$.

Lemma 3.1. Let $E$ be a compact subset of $\mathbb{A}_{\text {Berk, } \mathbb{C}_{v}}^{1}$ and let $U$ be the unbounded component of $\mathbb{A}_{\text {Berk, } \mathbb{C}_{v}}^{1} \backslash E$.

(1) If $\gamma(E)>0$ (i.e. $V(E)<\infty$ ), then $G_{E}(z)=V(E)+\log |z|_{v}$ for all $z \in \mathbb{A}_{\text {Berk, } \mathbb{C}_{v}}^{1}$ such that $|z|_{v}$ is sufficiently large.

(2) If $G_{E}(z)=0$ for all $z \in E$, then $G_{E}$ is continuous on $\mathbb{A}_{\text {Berk, } \mathbb{C}_{v}}^{1}, \operatorname{Supp}\left(\mu_{E}\right)=$ $\partial U$ and $G_{E}(z)>0$ if and only if $z \in U$.

(3) If $G: \mathbb{A}_{\text {Berk, } \mathbb{C}_{v}}^{1} \rightarrow \mathbb{R}$ is a continuous subharmonic function which is harmonic on $U$, identically zero on $E$, and such that $G(z)-\log ^{+}|z|_{v}$ is bounded, then $G=G_{E}$. Furthermore, if $G(z)=\log |z|_{v}+V+o(1)\left(\right.$ as $\left.|z|_{v} \rightarrow \infty\right)$ for some $V<\infty$, then $V(E)=V$ and so, $\gamma(E)=e^{-V}$.

To state the equidistribution result from [3], we consider the compact Berkovich adèlic sets which are of the following form

$$
\mathbb{E}:=\prod_{v \in \Omega} E_{v}
$$

where $E_{v}$ is a non-empty compact subset of $\mathbb{A}_{\text {Berk, } \mathbb{C}_{v}}^{1}$ for each $v \in \Omega$ and where $E_{v}$ is the closed unit disk $\mathcal{D}(0,1)$ in $\mathbb{A}_{\text {Berk, } \mathbb{C}_{v}}^{1}$ for all but finitely many $v \in \Omega$. The logarithmic capacity $\gamma(\mathbb{E})$ of $\mathbb{E}$ is defined as follows

$$
\gamma(\mathbb{E})=\prod_{v \in \Omega} \gamma\left(E_{v}\right)^{N_{v}}
$$

where the positive integers $N_{v}$ are the ones associated to the product formula on the global field $K$. Note that this is a finite product as for all but finitely many $v \in \Omega, \gamma\left(E_{v}\right)=\gamma(\mathcal{D}(0,1))=1$. Let $G_{v}=G_{E_{v}}$ be the Green's function of $E_{v}$ relative to $\infty$ for each $v \in \Omega$. For every $v \in \Omega$, we fix an embedding $\bar{K} \hookrightarrow \mathbb{C}_{v}$. Let $S \subset \bar{K}$ be any finite subset that is invariant under the action of the Galois group $\operatorname{Gal}(\bar{K} / K)$. We define the height $h_{\mathbb{E}}(S)$ of $S$ relative to $\mathbb{E}$ by

$$
h_{\mathbb{E}}(S)=\sum_{v \in \Omega} N_{v}\left(\frac{1}{|S|} \sum_{z \in S} G_{v}(z)\right) .
$$

Note that this definition is independent of any particular embedding $\bar{K} \hookrightarrow \mathbb{C}_{v}$ that we choose at $v \in \Omega$. The following is a special case of the equidistribution result [3, Theorem 7.52] that we need for our application.

Theorem 3.3. Let $\mathbb{E}=\prod_{v \in \Omega} E_{v}$ be a compact Berkovich adelic set with $\gamma(\mathbb{E})=$ 1. Suppose that $S_{n}$ is a sequence of $\operatorname{Gal}(\bar{K} / K)$-invariant finite subsets of $\bar{K}$ with $\left|S_{n}\right| \rightarrow \infty$ and $h_{\mathbb{E}}\left(S_{n}\right) \rightarrow 0$ as $n \rightarrow \infty$. For each $v \in \Omega$ and for each $n$ let $\delta_{n}$ be the discrete probability measure supported equally on the elements of $S_{n}$. Then the sequence of measures $\left\{\delta_{n}\right\}$ converges weakly to $\mu_{v}$ the equilibrium measure on $E_{v}$. 


\section{The Dynamics of the Drinfeld Module family $\Phi$}

Let $K$ be a finitely generated field extension of $\mathbb{F}_{q}(t)$. We work with a family of Drinfeld modules $\Phi$ as given in Theorem 2.4 , i.e.

$$
\Phi_{t}(x)=t x+\sum_{i=1}^{r-1} g_{i}(z) x^{q^{i}}+x^{q^{r}}
$$

with $g_{i}(z) \in K[z]$ for $i=1, \ldots, r-1$. For convenience, we let $g_{0}(z):=t$ be the corresponding constant polynomial. Let $\mathbf{c} \in K[z]$ be given. We define $f_{\mathbf{c}, n}(z):=$ $\Phi_{t^{n}}(\mathbf{c})$ for each $n \in \mathbb{N}$. Note that $f_{\mathbf{c}, n}$ is a polynomial in $z$ with coefficients in $K$. Assume $m:=\operatorname{deg}(\mathbf{c})$ satisfies the inequality

$$
m=\operatorname{deg}(\mathbf{c})>\max _{i=0}^{r-1} \frac{\operatorname{deg}\left(g_{i}\right)}{q^{r}-q^{i}} .
$$

We let $C_{m}$ be the leading coefficient of c. In the next Lemma we compute the degrees of all polynomials $f_{\mathbf{c}, n}$ for all positive integers $n$.

Lemma 4.2. With the above hypothesis, the polynomial $f_{\mathbf{c}, n}(z)$ has degree $m \cdot q^{r n}$ and leading coefficient $C_{m}^{q^{r n}}$ for each $n \in \mathbb{N}$.

Proof. The assertion follows easily by induction on $n$, using (4.1), since the term of highest degree in $z$ from $f_{\mathbf{c}, n}(z)$ is $\mathbf{c}^{q^{r n}}$.

We immediately obtain as a corollary of Lemma 4.2 the fact that $\mathbf{c}$ is not preperiodic for $\mathbf{f}$. Furthermore we obtain that if $\mathbf{c}(\lambda) \in \Phi_{\text {tor }}^{\lambda}$, then $\lambda \in \bar{K}$.

Fix a place $v \in \Omega_{K}$. Our first task is to define the generalized Mandelbrot set $M_{\mathbf{c}, v}$ associated to $\mathbf{c}$ and establish that $M_{\mathbf{c}, v}$ is a compact subset of $\mathbb{A}_{\text {Berk, }}^{1} \mathbb{C}_{v}$. Roughly speaking, $M_{\mathbf{c}, v}$ is the subset of $\mathbb{C}_{v}$ consisting of all $\lambda \in \mathbb{C}_{v}$ such that $\mathbf{c}(\lambda)$ is $v$-adic bounded under the action of $\mathbb{F}_{q}[t]$ with respect to the Drinfeld module structure $\Phi^{\lambda}$. A simple observation is that the orbit of $\mathbf{c}(\lambda)$ under the action of $\mathbb{F}_{q}[t]$ is $v$-adic bounded if and only if under the action of $\Phi_{t}^{\lambda}$ the orbit $\left\{\Phi_{t^{n}}^{\lambda}(\mathbf{c}(\lambda)) \mid n=0,1,2, \ldots\right\}$ is $v$-adic bounded. Hence, in our definition for the generalized Mandelbrot set $M_{\mathbf{c}, v}$ below we shall only consider the orbit of $\mathbf{c}(\lambda)$ under the action of $\Phi_{t}^{\lambda}$. Following [1. 16, we put

$$
M_{\mathbf{c}, v}:=\left\{\lambda \in \mathbb{A}_{\operatorname{Berk}, \mathbb{C}_{v}}^{1}: \sup _{n}\left[f_{\mathbf{c}, n}(T)\right]_{\lambda}<\infty\right\}
$$

Let $\lambda \in \mathbb{C}_{v}$ and recall the local canonical height $\widehat{\mathrm{h}}_{\Phi^{\lambda}, v}(x)$ of $x \in \mathbb{C}_{v}$ is given by the formula

$$
\widehat{\mathrm{h}}_{\Phi^{\lambda}, v}(x)=\lim _{n \rightarrow \infty} \frac{\log ^{+}\left|\Phi_{t^{n}}^{\lambda}(x)\right|_{v}}{q^{r n}} .
$$

Notice that $\widehat{\mathrm{h}}_{\Phi^{\lambda}, v}(x)$ is a continuous function of both $\lambda$ and $x$. As $\mathbb{C}_{v}$ is a dense subspace of $\mathbb{A}_{\text {Berk, } \mathbb{C}_{v}}^{1}$, continuity in $\lambda$ implies that the canonical local height function $\widehat{\mathrm{h}}_{\Phi^{\lambda}, v}(\mathbf{c}(\lambda))$ has a natural extension on $\mathbb{A}_{\text {Berk, } \mathbb{C}_{v}}^{1}$ (note that the topology on $\mathbb{C}_{v}$ is the restriction of the weak topology on $\mathbb{A}_{\text {Berk, }}^{1} \mathbb{C}_{v}$, so any continuous function on $\mathbb{C}_{v}$ will automatically have a unique extension to $\mathbb{A}_{\text {Berk, } \mathbb{C}_{v}}^{1}$ ). In the following, we will extend $\widehat{\mathrm{h}}_{\Phi^{\lambda}, v}(\mathbf{c}(\lambda))$ to a function of $\lambda$ on $\mathbb{A}_{\text {Berk, } \mathbb{C}_{v}}^{1}$ and view it as a continuous function on $\mathbb{A}_{\text {Berk, } \mathbb{C}_{v}}^{1}$. It follows from the definition of $M_{\mathbf{c}, v}$ that $\lambda \in M_{\mathbf{c}, v}$ if and only if $\widehat{\mathrm{h}}_{\Phi^{\lambda}, v}(\mathbf{c}(\lambda))=0$. Thus, $M_{\mathbf{c}, v}$ is a closed subset of $\mathbb{A}_{\text {Berk, } \mathbb{C}_{v}}^{1}$. In fact, the following is true. 
Proposition 4.3. $M_{\mathbf{c}, v}$ is a compact subset of $\mathbb{A}_{\text {Berk, } \mathbb{C}_{v}}^{1}$.

We already showed that $M_{\mathbf{c}, v}$ is a closed subset of the locally compact space $\mathbb{A}_{\text {Berk, } \mathbb{C}_{v}}^{1}$, and thus in order to prove Proposition 4.3 we only need to show that $M_{\mathbf{c}, v}$ is a bounded subset of $\mathbb{A}_{\text {Berk, }, \mathbb{C}_{v}}^{1}$.

Lemma 4.4. $M_{\mathbf{c}, v}$ is a bounded subset of $\mathbb{A}_{\text {Berk, }, \mathbb{C}_{v}}^{1}$.

Proof. For each $i=0, \ldots, r-1$, we let $D_{i}$ be the leading coefficient of $g_{i}$ (recall that $\left.g_{0}=t\right)$; also, let $d_{i}:=\operatorname{deg}\left(g_{i}\right)$. Using an argument identical as in deriving (2.2), we see that there exists $M>1$ such that if $|\lambda|_{v}>M$, then

$$
\begin{gathered}
\left|g_{i}(\lambda)\right|_{v}=\left|D_{i}\right|_{v} \cdot|\lambda|_{v}^{d_{i}} \text { for each } i=0, \ldots, r-1, \text { and } \\
|\mathbf{c}(\lambda)|_{v}=\left|C_{m}\right|_{v} \cdot|\lambda|_{v}^{m}>|\lambda|_{v}>1 .
\end{gathered}
$$

At the expense of replacing $M$ with a larger number we may assume that

$$
M^{m\left(q^{r}-q^{i}\right)-d_{i}} \geq\left|D_{i}\right|_{v} \cdot\left|C_{m}\right|_{v}^{q^{i}-q^{r}} \text { for each } i=0, \ldots, r-1 .
$$

Note that we may achieve the above inequality for large $M$ since (by our assumption (4.1) $), m>d_{i} /\left(q^{r}-q^{i}\right)$ for each $i=0, \ldots, r-1$. Therefore, if $|\lambda|_{v}>M$, then

$$
|\mathbf{c}(\lambda)|_{v}^{q^{r}}>\left|g_{i}(\lambda) \cdot \mathbf{c}(\lambda)^{q^{i}}\right|_{v} \text { for each } i=0, \ldots, r-1 .
$$

Hence,

$$
\left|\Phi_{t}^{\lambda}(\mathbf{c}(\lambda))\right|_{v}=|\mathbf{c}(\lambda)|_{v}^{q^{r}}>|\mathbf{c}(\lambda)|_{v}>|\lambda|_{v}>1 .
$$

This allows us to conclude that if $|\lambda|_{v}>M$, then $\mid \Phi_{t^{n}}^{\lambda}\left(\left.\mathbf{c}(\lambda)\right|_{v} \rightarrow \infty\right.$ as $n \rightarrow \infty$. Thus $\lambda \notin M_{\mathbf{c}, v}$ if $|\lambda|_{v}>M$.

Next our goal is to compute the logarithmic capacities of the $v$-adic generalized Mandelbrot sets $M_{\mathbf{c}, v}$ associated to $\mathbf{c}$ for the given family of Drinfeld module $\Phi$.

Theorem 4.5. The logarithmic capacity of $M_{\mathbf{c}, v}$ is $\gamma\left(M_{\mathbf{c}, v}\right)=\left|C_{m}\right|_{v}^{-1 / m}$.

The strategy for the proof of Theorem 4.5 is to construct a continuous subharmonic function $G_{c, v}: \mathbb{A}_{\text {Berk, } \mathbb{C}_{v}}^{1} \rightarrow \mathbb{R}$ satisfying Lemma 3.1 (3). We let

$$
G_{\mathbf{c}, v}(\lambda):=\lim _{n \rightarrow \infty} \frac{1}{\operatorname{deg}\left(f_{\mathbf{c}, n}\right)} \log ^{+}\left[f_{\mathbf{c}, n}(T)\right]_{\lambda} .
$$

Then by a similar reasoning as in the proof of [1, Prop. 3.7], it can be shown that the limit exists for all $\lambda \in \mathbb{A}_{\text {Berk, } \mathbb{C}_{v}}^{1}$. In fact, by the definition of canonical local height, for $\lambda \in \mathbb{C}_{v}$ we have

$$
\begin{aligned}
G_{\mathbf{c}, v}(\lambda) & =\lim _{n \rightarrow \infty} \frac{1}{m q^{r n}} \log ^{+}\left|f_{\lambda}^{n}(\mathbf{c}(\lambda))\right|_{v} \quad \text { since } \operatorname{deg}\left(f_{\mathbf{c}, n}\right)=m q^{r n} \text { by Lemma 4.2. } \\
& =\frac{1}{m} \cdot \widehat{\mathrm{h}}_{\Phi^{\lambda}, v}(\mathbf{c}(\lambda)) \quad \text { by the definition of canonical local height. }
\end{aligned}
$$

Note that $G_{\mathbf{c}, v}(\lambda) \geq 0$ for all $\lambda \in \mathbb{A}_{\text {Berk, } \mathbb{C}_{v}}^{1}$. Moreover, by definition we see that $\lambda \in M_{\mathbf{c}, v}$ if and only if $G_{\mathbf{c}, v}(\lambda)=0$.

Lemma 4.7. $G_{\mathbf{c}, v}$ is the Green's function for $M_{\mathbf{c}, v}$ relative to $\infty$.

The proof is essentially the same as the proof of [1, Prop. 3.7], we simply give a sketch of the idea. 
Proof of Lemma 4.7. So, using the same argument as in the proof of [7, Prop. 1.2], we observe that as a function of $\lambda$, the function $\frac{\log ^{+}\left[f_{\mathbf{c}, n}(T)\right]_{\lambda}}{\operatorname{deg}\left(f_{\mathbf{c}, n}\right)}$ converges uniformly on compact subsets of $\mathbb{A}_{\text {Berk, } \mathbb{C}_{v}}^{1}$. So, the function $\frac{\log ^{+}\left[f_{\mathbf{c}, n}(T)\right]_{\lambda}}{\operatorname{deg}\left(f_{\mathbf{c}, n}\right)}$ is a continuous subharmonic function on $\mathbb{A}_{\text {Berk, }, \mathbb{C}_{v}}^{1}$, which converges to $G_{\mathbf{c}, v}$ uniformly; hence it follows from [3, Prop. 8.26(c)] that $G_{\mathbf{c}, v}$ is continuous and subharmonic on $\mathbb{A}_{\text {Berk, } \mathbb{C}_{v}}^{1}$. Furthermore, as remarked above, $G_{\mathbf{c}, v}$ is zero on $M_{\mathbf{c}, v}$.

Arguing as in the proof of Lemma 4.4, if $|\lambda|_{v}$ is sufficiently large, then for $n \geq 1$ we have

$$
\left|f_{\mathbf{c}, n}(\lambda)\right|_{v}=\left|\Phi_{t^{n}}^{\lambda}(\mathbf{c}(\lambda))\right|_{v}=\left|C_{m} \lambda^{m}\right|_{v}^{q^{r n}} .
$$

Hence, for $|\lambda|_{v}$ sufficiently large we have

$$
\begin{aligned}
G_{\mathbf{c}, v}(\lambda) & =\lim _{n \rightarrow \infty} \frac{1}{m q^{r n}} \log \left|f_{\mathbf{c}, n}(\lambda)\right|_{v} \\
& =\log |\lambda|_{v}+\frac{\log \left|C_{m}\right|_{v}}{m} .
\end{aligned}
$$

It follows from Lemma 3.1(3), that $G_{\mathbf{c}, v}$ is indeed the Green's function of $M_{\mathbf{c}, v}$.

Now we are ready to prove Theorem 4.5 .

Proof of Theorem 4.5. As in the proof of Lemma 4.7, we have

$$
G_{\mathbf{c}, v}(\lambda)=\log |\lambda|_{v}+\frac{\log \left|C_{m}\right|_{v}}{m}
$$

for $|\lambda|_{v}$ sufficiently large. By Lemma 3.1 (3), we find that $V\left(M_{\mathbf{c}, v}\right)=\frac{\log \left|C_{m}\right|_{v}}{m}$. Hence, the logarithmic capacity of $M_{\mathbf{c}, v}$ is

$$
\gamma\left(M_{\mathbf{c}, v}\right)=e^{-V\left(M_{\mathbf{c}, v}\right)}=\frac{1}{\left|C_{m}\right|_{v}^{1 / m}}
$$

as desired.

Let $\mathbb{M}_{\mathbf{c}}=\prod_{v \in \Omega} M_{\mathbf{c}, v}$ be the generalized adèlic Mandelbrot set associated to $c$. As a corollary to Theorem 4.5 we see that $\mathbb{M}_{\mathbf{c}}$ satisfies the hypothesis of Theorem 3.3 .

Corollary 4.8. For all but finitely many nonarchimedean places $v$, we have that $M_{\mathbf{c}, v}$ is the closed unit disk $\mathcal{D}(0 ; 1)$ in $\mathbb{A}_{\text {Berk, }, \mathbb{C}_{v}} ;$ furthermore $\gamma\left(\mathbb{M}_{\mathbf{c}}\right)=1$.

Proof. For each place $v$ where all coefficients of $g_{i}(z), i=0, \ldots, r-1$ and of $\mathbf{c}(z)$ are $v$-adic integral, and moreover $\left|C_{m}\right|_{v}=1$, we have that $M_{\mathbf{c}, v}=\mathcal{D}(0,1)$. Indeed, $\mathcal{D}(0,1) \subset M_{\mathbf{c}, v}$ since then $\Phi_{t^{n}}^{\lambda}(\mathbf{c}(\lambda))$ is always a $v$-adic integer. For the converse implication we note that each coefficient of $f_{\mathbf{c}, n}(z)$ is a $v$-adic integer, while the leading coefficient is a $v$-adic unit for all $n \geq 1$; thus $\left|f_{\mathbf{c}, n}(\lambda)\right|_{v}=|\lambda|_{v}^{m q^{r n}} \rightarrow \infty$ if $|\lambda|_{v}>1$. Note that $C_{m} \neq 0$ and so, the second assertion in Corollary 4.8 follows immediately by the product formula in $K$.

Using the decomposition of the global canonical height as a sum of local canonical heights we obtain the following result.

Corollary 4.9. Let $\lambda \in \bar{K}$, let $S$ be the set of $\operatorname{Gal}(\bar{K} / K)$-conjugates of $\lambda$, and let $h_{\mathbb{M}_{\mathbf{c}}}$ be defined as in (3.2). Then $\operatorname{deg}(\mathbf{c}) \cdot h_{\mathbb{M}_{\mathbf{c}}}(S)=\widehat{\mathrm{h}}_{\Phi^{\lambda}}(\mathbf{c}(\lambda))$. 
Remark 4.10. Corollary 4.9 is a result on specialization of height functions relating the canonical heights of $\mathbf{c}(\lambda)$ in the family and the height of the parameter $\lambda$. As a consequence of Corollary 4.9, we find that $\mathbf{c}(\lambda)$ is a torsion point for $\Phi^{\lambda}$ if and only if $h_{\mathbb{M}_{\mathbf{c}}}(S)=0$.

By abuse of notation we will use the notation $h_{\mathbb{M}_{\mathbf{c}}}(\lambda):=h_{\mathbb{M}_{\mathbf{c}}}(S)$ where $S$ is the $\operatorname{Gal}(\bar{K} / K)$-orbit of $\lambda$.

\section{Proof of Theorem 2.4}

We work under the hypothesis of Theorem 2.4, and we continue with the notation from the previous Sections.

Recall that $\Phi_{t}(x)=t x+\sum_{i=0}^{r-1} g_{i}(z) x^{q^{i}}+x^{q^{r}}$ where we require that $g_{i} \in K[z]$ for $i=1, \ldots, r-1$. Let $\mathbf{a}, \mathbf{b} \in K[z]$ satisfying the hypothesis (2.5) of Theorem [2.4] In particular, both $\mathbf{a}$ and $\mathbf{b}$ have positive degrees. At the expense of replacing $K$ with the extension of $\mathbb{F}_{q}(t)$ generated by all coefficients of $g_{i}$, and of $\mathbf{a}$ and of $\mathbf{b}$, we may assume that $K$ is finitely generated over $\mathbb{F}_{q}(t)$. Let $\Omega_{K}$ be the set of all inequivalent absolute values on $K$ constructed as in Subsection 2.2 ,

Next, assume there exist infinitely many $\lambda$ such that $\mathbf{a}(\lambda), \mathbf{b}(\lambda) \in \Phi_{\text {tor }}^{\lambda}$. As a consequence of Lemma 4.2, we have $\lambda \in \bar{K}$.

Let $h_{\mathbb{M}_{\mathbf{a}}}(z)\left(h_{\mathbb{M}_{\mathbf{b}}}(z)\right)$ be the height of $z \in \bar{K}$ relative to the adelic generalized Mandelbrot set $\mathbb{M}_{\mathbf{a}}:=\prod_{v \in \Omega_{K}} M_{\mathbf{a}, v}$ (respectively, $\mathbb{M}_{\mathbf{b}}$ ) defined as in Section 4 (see also Remark 4.10). Note that if $\lambda \in \bar{K}$ is a parameter such that $\mathbf{a}(\lambda)$ (and $\mathbf{b}(\lambda))$ is torsion for $\Phi^{\lambda}$ then $h_{\mathbb{M}_{\mathbf{a}}}(\lambda)=0$ by Corollary 4.9. So, we may apply the equidistribution result from Theorem 3.3 and conclude that $M_{\mathbf{a}, v}=M_{\mathbf{b}, v}$ for each place $v \in \Omega_{K}$. Indeed, we know that there exists an infinite sequence $\left\{\lambda_{n}\right\}_{n \in \mathbb{N}}$ of distinct numbers $\lambda \in \bar{K}$ such that both $\mathbf{a}(\lambda)$ and $\mathbf{b}(\lambda)$ are torsion points for $\Phi^{\lambda}$. So, for each $n \in \mathbb{N}$, we may take $S_{n}$ be the union of the sets of Galois conjugates for $\lambda_{m}$ for all $1 \leq m \leq n$. Clearly $\# S_{n} \rightarrow \infty$ as $n \rightarrow \infty$, and also each $S_{n}$ is $\operatorname{Gal}(\bar{K} / K)$ invariant. Finally, $h_{\mathbb{M}_{\mathbf{a}}}\left(S_{n}\right)=h_{\mathbb{M}_{\mathbf{b}}}\left(S_{n}\right)=0$ for all $n \in \mathbb{N}$, and thus Theorem 3.3 applies in this case. We obtain that $\mu_{\mathbb{M}_{\mathfrak{a}}}=\mu_{\mathbb{M}_{\mathrm{b}}}$ and since they are both supported on $\mathbb{M}_{\mathbf{a}}$ (resp. $\mathbb{M}_{\mathbf{b}}$ ), we also get that $\mathbb{M}_{\mathbf{a}}=\mathbb{M}_{\mathbf{b}}$. Consequently, the two height functions $h_{\mathbb{M}_{\mathbf{a}}}$ and $h_{\mathbb{M}_{\mathbf{b}}}$ are equal. Using Corollary 4.9 again, we conclude that for each $\lambda \in \bar{K}$ we have that $\widehat{\mathrm{h}}_{\Phi^{\lambda}}(\mathbf{a}(\lambda))=0$ if and only if $\widehat{\mathrm{h}}_{\Phi^{\lambda}}(\mathbf{b}(\lambda))=0$. Hence $\mathbf{a}(\lambda) \in \Phi_{\text {tor }}^{\lambda}$ if and only if $\mathbf{b}(\lambda) \in \Phi_{\text {tor }}^{\lambda}$.

Finally, knowing that $\mathbb{M}_{\mathbf{a}}=\mathbb{M}_{\mathbf{b}}$ we obtain that the capacities of the corresponding generalized Mandelbrot sets are equal to each other, i.e.,

$$
\left|C_{\mathbf{a}}\right|^{\frac{1}{\operatorname{deg}(\mathbf{a})}}=\left|C_{\mathbf{b}}\right|_{v}^{\frac{1}{\operatorname{deg}(\mathbf{b})}} \text { for each place } v \text {. }
$$

We let $U:=C_{\mathbf{a}}^{\operatorname{deg}(\mathbf{b})} / C_{\mathbf{b}}^{\operatorname{deg}(\mathbf{a})}$. Then for each $v \in \Omega_{K}$ we know that $|U|_{v}=1$. Since the only elements of $K$ which are units for all places $v \in \Omega_{K}$ are the ones living in $\overline{\mathbb{F}_{q}}$, we conclude that $U \in \overline{\mathbb{F}_{q}}$, as desired.

\section{Proof of our MAin RESUlts}

Theorem 1.3 follows from Theorem 2.4 .

Proof of Theorem 1.3. If either $\mathbf{a}$ or $\mathbf{b}$ is a torsion point for $\Phi$, then we are done. So, from now on, assume that neither $\mathbf{a}$ nor $\mathbf{b}$ is torsion for $\Phi$.

We now consider the given Drinfeld module $\Phi: \mathbb{F}_{q}[t] \longrightarrow \operatorname{End}_{L}\left(\mathbb{G}_{a}\right)$ as a Drinfeld module over $L=K(z)$ the rational function field with constant field $K$. We let $\Omega_{L / K}$ 
be the set of all places of the function field $L / K$, and let $\widehat{\mathrm{h}}_{\Phi}$ be the canonical height for the Drinfeld module $\Phi$ over $L$. There are two cases.

Case 1. Each polynomial $g_{i}$ is constant.

In this case, we have that $\Phi_{\text {tor }}^{\lambda}=\Phi_{\text {tor }} \subset \bar{K}$. Since $\mathbf{a}$ and $\mathbf{b}$ are not torsion for $\Phi$, we conclude that $\mathbf{a}$ (and similarly $\mathbf{b}$ ) is not a constant polynomial, otherwise $\mathbf{a} \notin \Phi_{\text {tor }}^{\lambda}$ (b $\notin \Phi_{\text {tor }}^{\lambda}$, respectively) for all $\lambda \in \bar{K}$. This contradicts our hypothesis that there exist infinitely many $\lambda \in \bar{K}$ such that $\mathbf{a}(\lambda)$ and $\mathbf{b}(\lambda)$ are torsion for $\Phi^{\lambda}$. Therefore, according to [15, Theorem 4.15 (a)], there exists $w \in \Omega_{L / K}$ such that $\widehat{\mathrm{h}}_{\Phi, w}(\mathbf{a})>0$ (a similar statement holds for $\mathbf{b}$ ).

Case 2. There exists at least one polynomial $g_{i}$ which is not constant.

In this case, there exists at least one place $v \in \Omega_{L / K}$ such that for some $i=$ $1, \ldots, r-1$, we have $\left|g_{i}\right|_{v}>1$ and thus [15, Theorem 4.15 (b)] yields that there exists some place $w \in \Omega_{L / K}$ such that $\widehat{\mathrm{h}}_{\Phi, w}(\mathbf{a})>0$. A similar statement holds for $\mathbf{b}$ since we know that neither $\mathbf{a}$ nor $\mathbf{b}$ is torsion for $\Phi$.

Therefore we know that there exists some place $w \in \Omega_{L / K}$ such that $\widehat{\mathrm{h}}_{\Phi, w}(\mathbf{a})>0$, i.e.,

$$
\left|\Phi_{t^{n}}(\mathbf{a})\right|_{w} \rightarrow \infty \text { as } n \rightarrow \infty .
$$

However, since $\mathbf{a} \in K[z]$ and each $g_{i} \in K[z]$ and also $t \in K$ we obtain that for each place $v \in \Omega_{L / K}$ except the place at infinity, the iterates $\Phi_{t^{n}}(\mathbf{a})$ are all $v$-integral. Hence it must be that for the place $w$ at infinity of the function field $K(z)$ we have that (6.1) holds, i.e.

$$
\operatorname{deg}_{z}\left(\Phi_{t^{n}}(\mathbf{a})\right) \rightarrow \infty \text { as } n \rightarrow \infty .
$$

A similar argument yields that also $\operatorname{deg}_{z}\left(\Phi_{t^{n}}(\mathbf{b})\right) \rightarrow \infty$ as $n \rightarrow \infty$. Hence, at the expense of replacing both $\mathbf{a}$ and $\mathbf{b}$ by $\Phi_{t^{n}}(\mathbf{a})$ respectively $\Phi_{t^{n}}(\mathbf{b})$ (for a sufficiently large integer $n$ ), we may achieve that inequality (2.5) is satisfied. Moreover, note that for each $\lambda$, we have that $\mathbf{a}(\lambda)$ ( $\operatorname{or} \mathbf{b}(\lambda)$ ) is a torsion point for $\Phi^{\lambda}$ if and only if $\Phi_{t^{n}}^{\lambda}(\mathbf{a}(\lambda))$ (respectively $\Phi_{t^{n}}^{\lambda}(\mathbf{b}(\lambda))$ ) is a torsion point for $\Phi^{\lambda}$. Theorem 2.4 yields that for each $\lambda \in \bar{K}$ we have that $\Phi_{t^{n}}^{\lambda}(\mathbf{a}(\lambda))$ (and thus $\mathbf{a}(\lambda)$ ) is a torsion point for $\Phi^{\lambda}$ if and only if $\Phi_{t^{n}}^{\lambda}(\mathbf{b}(\lambda))$ (and thus $\mathbf{b}(\lambda)$ ) is a torsion point for $\Phi^{\lambda}$.

In the following, for a nonzero element $f \in \mathbb{F}_{q}[t]$ and a Drinfeld module $\phi$ we denote the submodule of $f$-torsion by $\phi[f]$ as usual.

Proof of Theorem 1.4. If either $\mathbf{a}$ or $\mathbf{b}$ equals 0 , then clearly $\mathbf{a}$ (or $\mathbf{b}$ ) is always torsion and the conclusion is immediate. So, assume now that both $\mathbf{a}$ and $\mathbf{b}$ are nonzero.

Assume first that $\mathbf{a}$ and $\mathbf{b}$ are $\mathbb{F}_{q}$-linearly dependent. We note that for each positive integer $n$ we have then that $\Phi_{t^{n}}(\mathbf{a})$ is a polynomial of degree $q^{r n}$ in $z$. On the other hand, for any two distinct, monic, irreducible polynomials $f, g \in \mathbb{F}_{p}[t]$ we have $\Phi[f] \cap \Phi[g]=\{0\}$. Therefore by solving $\Phi_{f}^{\lambda}(\mathbf{a})=0$ for various distinct, monic, irreducible polynomials $f \in \mathbb{F}_{q}[t]$ we find that there exist infinitely many $\lambda \in \bar{K}$ such that $\mathbf{a} \in \Phi_{\text {tor }}^{\lambda}$. On the other hand, for any $c \in \mathbb{F}_{q}$ and $a \in \mathbb{F}_{q}[t]$ we have that $c \Phi_{a}^{\lambda}(x)=\Phi_{a}^{\lambda}(c x)$. From this, it is easy to see that for each $\lambda \in \bar{K}$ we have that $\mathbf{a}$ is a torsion point if and only if $\mathbf{b}=c \mathbf{a}\left(c \in \mathbb{F}_{q}\right)$ is a torsion point for $\Phi^{\lambda}$. So, indeed there exist infinitely many $\lambda \in \bar{K}$ such that $\mathbf{a}, \mathbf{b} \in \Phi_{\text {tor }}^{\lambda}$.

Now for the converse, we note that

$$
\mathbf{a}_{1}:=\Phi_{t^{2}}(\mathbf{a})=\Phi_{t}\left(\mathbf{a}^{q} \cdot z+\left(t \mathbf{a}+\mathbf{a}^{q^{r}}\right)\right)
$$


and

$$
\mathbf{b}_{1}:=\Phi_{t^{2}}(\mathbf{b})=\Phi_{t}\left(\mathbf{b}^{q} \cdot z+\left(t \mathbf{b}+\mathbf{b}^{q^{r}}\right)\right)
$$

are both polynomials in $z$ of same degree $q^{r}>1$. Furthermore their leading coefficients are $\mathbf{a}^{q^{r+1}}$, respectively $\mathbf{b}^{q^{r+1}}$. Therefore inequality (2.5) from Theorem 2.4 is satisfied and thus we conclude that $\mathbf{a} / \mathbf{b} \in \overline{\mathbb{F}_{p}}$. On the other hand we know that $\mathbf{a}, \mathbf{b} \in K$ and $\mathbb{F}_{q}$ is algebraically closed in $K$; hence $\mathbf{a} / \mathbf{b} \in \mathbb{F}_{q}$ as desired.

Similarly to Theorem 1.4 we can prove Theorem 1.5 .

Proof of Theorem 1.5. Let $s$ be a positive integer such that $\mathbf{a}, \mathbf{b} \in \mathbb{F}_{q^{s}}(t)$ and let $\Omega_{s}:=\Omega_{\mathbb{F}_{q^{s}}(t)}$. Arguing as in the proof of Theorem 1.4 we obtain that if $\mathbf{a}$ and b are $\mathbb{F}_{q}$-linearly dependent, then there exist infinitely many $\lambda \in \overline{\mathbb{F}_{p}(t)}$ such that $\mathbf{a}, \mathbf{b} \in \Phi_{\text {tor }}^{\lambda}$.

Assume now that there exist infinitely many $\lambda \in \overline{\mathbb{F}_{q}(t)}$ such that $\mathbf{a}, \mathbf{b} \in \Phi_{\text {tor }}^{\lambda}$. In addition, we may assume both $\mathbf{a}$ and $\mathbf{b}$ are nonzero. The proof of Theorem 1.4 shows that $\mathbf{a} / \mathbf{b} \in \overline{\mathbb{F}_{q}}$. In addition, Theorem 2.4 yields that for each $\lambda \in \overline{\mathbb{F}_{q}(t)}$, we have that $\mathbf{a} \in \Phi_{\text {tor }}^{\lambda}$ if and only if $\mathbf{b} \in \Phi_{\text {tor. }}^{\lambda}$. In order to finish the proof of Theorem 1.5 we will use both consequences of Theorem 2.4 stated above.

We let $\gamma:=\mathbf{b} / \mathbf{a} \in \mathbb{F}_{q^{s}}$, and assume $\gamma \notin \mathbb{F}_{q}$. Let $\lambda_{0} \in \overline{\mathbb{F}_{q}(t)}$ such that $\Phi_{t}^{\lambda_{0}}(\mathbf{a})=0$. Then, we have

$$
\lambda_{0} \mathbf{a}^{q}=-t \mathbf{a}-\mathbf{a}^{q^{r}} .
$$

We will show that $\mathbf{b} \notin \Phi_{\text {tor }}^{\lambda_{0}}$ which yields a contradiction to the conclusion of Theorem 2.4 Before we proceed, we note that

$$
\begin{aligned}
\Phi_{t}^{\lambda_{0}}(\mathbf{b}) & =\gamma t \mathbf{a}+\gamma^{q} \lambda_{0} \mathbf{a}^{q}+\gamma^{q^{2}} \mathbf{a}^{q^{2}} \\
& =\left(\gamma-\gamma^{q}\right) t \mathbf{a}+\left(\gamma^{q^{r}}-\gamma^{q}\right) \mathbf{a}^{q^{r}} \text { by (6.2). }
\end{aligned}
$$

In the following, we denote by $|\cdot|_{\infty}$ the absolute value corresponding to the unique place of $\mathbb{F}_{q^{s}}(t)$ where $t$ is not integral. We split our analysis into two cases:

Case 1. a $\in \mathbb{F}_{q^{s}}$.

By (6.2) we have that $\left|\lambda_{0}\right|_{\infty}=|t|_{\infty}$ and it follows from (6.3) that

$\left|\Phi_{t}^{\lambda_{0}}(\mathbf{b})\right|_{\infty}=\left|\left(\gamma-\gamma^{q}\right) t \mathbf{a}+\left(\gamma^{q^{r}}-\gamma^{q}\right) \mathbf{a}^{q^{r}}\right|_{\infty}=|t|_{\infty}>\max \left\{1,|t|_{\infty}^{1 /\left(q^{r}-1\right)},\left|\lambda_{0}\right|_{\infty}^{1 /\left(q^{r}-q\right)}\right\}$.

Using (2.2), we conclude that $\left|\Phi_{t^{n}}^{\lambda_{0}}(\mathbf{b})\right|_{\infty} \rightarrow \infty$ as $n \rightarrow \infty$ and hence $\mathbf{b} \notin \Phi_{\text {tor }}^{\lambda_{0}}$ as desired.

Case 2. a $\notin \mathbb{F}_{q^{s}}$.

In this case there exists a place $v \in \Omega_{s}$ such that $|\mathbf{a}|_{v}>1$. Note that it is not possible to have $|t \mathbf{a}|_{v} \geq|\mathbf{a}|^{q^{r}}$ for otherwise also $|t|_{v}>1$. This implies that $|\cdot|_{v}=|\cdot|_{\infty}$ and $|t|_{\infty} \geq|\mathbf{a}|_{\infty}^{q^{r}-1}$. But this is impossible in $\mathbb{F}_{q^{s}}(t)$. Hence, $|t \mathbf{a}|_{v}<|\mathbf{a}|_{v}^{q^{r}}$ and so, $\left|\lambda_{0}\right|_{v}=|\mathbf{a}|_{v}^{q^{r}-q}$.

There are two possibilities now.

Case 2a. $\gamma^{q^{r}}-\gamma^{q} \neq 0$.

Consequently, we have

$$
\left|\Phi_{t}^{\lambda_{0}}(\mathbf{b})\right|_{v}=\max \left\{|t \mathbf{a}|_{v},|\mathbf{a}|_{v}^{q^{r}}\right\}=|\mathbf{a}|_{v}^{q^{r}} .
$$

It follows that

$$
\text { - }|t|_{v}^{1 /\left(q^{r}-1\right)} \leq|\mathbf{a}|_{v},
$$


- $\left|\lambda_{0}\right|_{v}^{1 /\left(q^{r}-q\right)}=|\mathbf{a}|_{v}$ and

- $\left|\Phi_{t}^{\lambda_{0}}(\mathbf{b})\right|_{v}=|\mathbf{a}|_{v}^{q^{r}}>|\mathbf{a}|_{v}=\max \left\{1,|t|_{v}^{1 /\left(q^{r}-1\right)},\left|\lambda_{0}\right|_{v}^{1 /\left(q^{r}-q\right)}\right\}$.

Using (2.2) again, we conclude that $\left|\Phi_{t^{n}}^{\lambda_{0}}(\mathbf{b})\right|_{v} \rightarrow \infty$ as $n \rightarrow \infty$ and $\mathbf{b} \notin \Phi_{\text {tor }}^{\lambda_{0}}$.

Case 2b. $\gamma^{q^{r}}=\gamma^{q}$.

Since we assumed that $\gamma \notin \mathbb{F}_{q}$, then we obtain that $r \geq 3$. By (6.3) and the assumption in Case 2b we have that

$$
\Phi_{t}^{\lambda_{0}}(\mathbf{b})=\left(\gamma-\gamma^{q}\right) t \mathbf{a}
$$

If $|t|_{v}>1$ (i.e., $v$ is the place $\infty$ ) then $\left|\Phi_{t}^{\lambda_{0}}(\mathbf{b})\right|_{v}>|\mathbf{a}|_{v}$ and so, again (2.2) can be used to infer that $\left|\Phi_{t^{n}}^{\lambda_{0}}(\mathbf{b})\right|_{v} \rightarrow \infty$ since

$$
\left|\Phi_{t}^{\lambda_{0}}(\mathbf{b})\right|_{v}>|\mathbf{a}|_{v}=\max \left\{|t|_{v}^{1 /\left(q^{r}-1\right)},\left|\lambda_{0}\right|^{1 /\left(q^{r}-q\right)}\right\} .
$$

Assume now that $|t|_{v} \leq 1$. Using the assumption on $\gamma$ from Case $\mathbf{2} \mathbf{b}$ we get

$$
\begin{aligned}
\Phi_{t^{2}}^{\lambda_{0}}(\mathbf{b}) & =\left(\gamma-\gamma^{q}\right) t^{2} \mathbf{a}+\left(\gamma-\gamma^{q}\right)^{q} t^{q} \mathbf{a}^{q} \lambda_{0}+\left(\gamma-\gamma^{q}\right)^{q^{r}} t^{q^{r}} \mathbf{a}^{q^{r}} \\
& =\left(\gamma-\gamma^{q}\right) t^{2} \mathbf{a}+\left(\gamma^{q}-\gamma^{q^{2}}\right) t^{q} \mathbf{a}^{q} \lambda_{0}+\left(\gamma^{q}-\gamma^{q^{2}}\right) t^{q^{r}} \mathbf{a}^{q^{r}} \\
& =\left(\gamma-\gamma^{q}\right) t^{2} \mathbf{a}+\left(\gamma^{q}-\gamma^{q^{2}}\right) \cdot\left(t^{q} \mathbf{a}^{q} \lambda_{0}+t^{q^{r}} \mathbf{a}^{q^{r}}\right) \\
& =\left(\gamma-\gamma^{q}\right) t^{2} \mathbf{a}+\left(\gamma^{q}-\gamma^{q^{2}}\right) \cdot\left(-t^{q+1} \mathbf{a}-t^{q} \mathbf{a}^{q^{r}}+t^{q^{r}} \mathbf{a}^{q^{r}}\right) \text { using (6.2) } \\
& =\left(\gamma-\gamma^{q}\right) t^{2} \mathbf{a}+\left(\gamma^{q}-\gamma^{q^{2}}\right) \cdot\left(-t^{q+1} \mathbf{a}+\mathbf{a}^{q^{r}} \cdot\left(t^{q^{r}}-t^{q}\right)\right) .
\end{aligned}
$$

Since $|\mathbf{a}|_{v}>1$ and $v \in \Omega_{s}$ we conclude that

$$
\left|t^{q^{r}}-t^{q}\right|_{v}=\left|t^{q^{r-1}}-t\right|_{v}^{q} \geq|\mathbf{a}|_{v}^{-q}
$$

since $t^{q^{r-1}}-t$ is a separable polynomial and thus it is either a $v$-adic unit or has the $v$-adic absolute value equal to that of a uniformizer of $v$ in $\mathbb{F}_{q^{s}}(t)$ (note the assumption that $\left.|t|_{v} \leq 1\right)$. So, using (6.4) we get that

$$
\begin{aligned}
\left|\mathbf{a}^{q^{r}} \cdot\left(t^{q^{r}}-t^{q}\right)\right|_{v} & \geq|\mathbf{a}|_{v}^{q^{r}-q} \\
& \geq|\mathbf{a}|_{v}^{q^{3}-q} \text { because } r \geq 3 \text { in Case } \mathbf{2} \mathbf{b} \\
& >|\mathbf{a}|_{v} \text { because } q \geq 2 \\
& \geq\left|t^{q+1} \mathbf{a}\right|_{v} \text { because }|t|_{v} \leq 1 \text { by our assumption. }
\end{aligned}
$$

Therefore (using also that $\gamma \notin \mathbb{F}_{q}$ and thus $\gamma^{q}-\gamma^{q^{2}} \neq 0$ )

$$
\left|\left(\gamma^{q}-\gamma^{q^{2}}\right) \cdot\left(-t^{q+1} \mathbf{a}+\mathbf{a}^{q^{r}} \cdot\left(t^{q^{r}}-t^{q}\right)\right)\right|_{v} \geq|\mathbf{a}|_{v}^{q^{r}-q} .
$$

On the other hand

$$
\left|\left(\gamma-\gamma^{2}\right) t^{2} \mathbf{a}\right|_{v} \leq|\mathbf{a}|_{v}
$$

because $|t|_{v} \leq 1$. Using (6.5) and (6.6) coupled with the fact that

$$
q^{r}-q>1 \text { since } r \geq 3 \text { and } q \geq 2
$$

we conclude that

$$
\left|\Phi_{t^{2}}^{\lambda_{0}}(\mathbf{b})\right|_{v} \geq|\mathbf{a}|_{v}^{q^{r}-q}>|\mathbf{a}|_{v}=\max \left\{|t|_{v}^{1 /\left(q^{r}-1\right)},\left|\lambda_{0}\right|^{1 /\left(q^{r}-q\right)}\right\} .
$$


Again using (2.2) yields that $\left|\Phi_{t^{n}}^{\lambda_{0}}(\mathbf{b})\right|_{v} \rightarrow \infty$ as $n \rightarrow \infty$ and thus $\mathbf{b} \notin \Phi_{\text {tor }}^{\lambda_{0}}$.

In conclusion, assuming that $\gamma \notin \mathbb{F}_{q}$ yields in each case a contradiction; this finishes the proof of Theorem 1.5 .

\section{REFERENCES}

[1] M. Baker and L. DeMarco, Preperiodic points and unlikely intersections, Duke Math. J. 159 (2011), 1-29.

[2] M. Baker and R. Rumely, Equidistribution of small points, rational dynamics, and potential theory, Ann. Inst. Fourier (Grenoble) 56(3) (2006), 625-688.

[3] M. Baker and R. Rumely, Potential theory and dynamics on the Berkovich projective line, Mathematical Surveys and Monographs 159. American Mathematical Society, Providence, RI, 2010.

[4] V. Berkovich, Spectral theory and analytic geometry over non-Archimedean fields, Amer. Math. Soc., Providence (1990), x+169 pp.

[5] E. Bombieri, D. Masser, and U. Zannier, Intersecting a curve with algebraic subgroups of multiplicative groups, IMRN 20 (1999), 1119-1140.

[6] E. Bombieri and W. Gubler, Heights in Diophantine Geometry, New Mathematical Monograph, Cambridge Univ. Press, Cambridge 3 (2006),

[7] B. Branner and J. H. Hubbard, The iteration of cubic polynomials. I. The global topology of parameter space., Acta Math. 160(3-4) (1988), 143-206.

[8] G. S. Call and J. H. Silverman, Canonical heights on varieties with morphisms, Compositio Math. 89 (1993), 163-205.

[9] L. Carleson and T. W. Gamelin, Complex dynamics, Springer-Verlag, New York, 1993.

[10] A. Chambert-Loir, Mesures et équidistribution sur les espaces de Berkovich, J. Reine Angew. Math. 595 (2006), 215-235.

[11] A. J. de Jong, Smoothness, semi-stability and alterations, Inst. Hautes Études Sci. Publ. Math. No. 83 (1996), 51-93.

[12] L. Denis, Hauters canoniques et modules de Drinfeld, Math. Ann. 294 (1992), 213-223.

[13] C. Favre and J. Rivera-Letelier, Théorème d'équidistribution de Brolin en dynamique paddique, C. R. Math. Acad. Sci. Paris 339(4) (2004), 271-276.

[14] C. Favre and J. Rivera-Letelier, Équidistribution quantitative des points de petite hauteur sur la droite projective, Math. Ann. 355(2) (2006), 311-361.

[15] D. Ghioca, The Lehmer inequality and the Mordell-Weil theorem for Drinfeld modules, J. Number Theory 122 (2007), 37-68.

[16] D. Ghioca, L.-C. Hsia, and T. J. Tucker, Preperiodic points for families of polynomials, to appear in Algebra \& Number Theory (2012), 28 pages.

[17] D. Ghioca and T. J. Tucker, Equidistribution and integral points for Drinfeld modules, Trans. Amer. Math. Soc. 360 (2008), 4863-4887.

[18] D. Goss, Basic Structure of Function field Arithmetic, Springer-Verlag, Berlin, 1996.

[19] P. Habegger, Intersecting subvarieties of abelian varieties with algebraic subgroups of complementary dimension, Invent. Math. 176 (2009), 405-447.

[20] M. Hindry, Autour d'une conjecture de Serge Lang, Invent. Math. 94 (1988), 575-603.

[21] L.-C. Hsia, On the reduction of a non-torsion point of a Drinfeld module, J. Number Theory 128 (2008), 1458-1484.

[22] S. Lang, Division points on curves, Ann. Mat. Pura Appl. (4) 70 (1965), 229-234.

[23] S. Lang, Fundamental of Diophantine Geometry, Springer-Verlag, New York, 1983.

[24] D. Masser and U. Zannier, Torsion anomalous points and families of elliptic curves, Amer. J. Math. 132 (2010), 1677-1691.

[25] D. Masser and U. Zannier, Torsion points on families of squares of elliptic curves, to appear in Math. Ann., Article DOI: 10.1007/s00208-011-0645-4.

[26] R. Pink, A common generalization of the conjectures of André-Oort, Manin-Mumford, and Mordell-Lang, preprint, 2005.

[27] B. Poonen, Local height functions and the Mordell-Weil theorem for Drinfeld modules, Compositio Math. 97 (1995), 349-368.

[28] M. Raynaud, Courbes sur une variété abélienne et points de torsion, Invent. Math. 71 (1983), $207-233$. 
[29] M. Raynaud, Sous-variétés d'une variété abélienne et points de torsion, Arithmetic and Geometry, vol. I, 327-352. Progress in Mathematics 35. Boston, MA, Birkhäuser, 1983.

[30] J. T.-Y. Wang, The Mordell-Weil theorems for Drinfeld modules over finitely generated function fields, Manuscripta math. 106 (2001), 305-314.

Dragos Ghioca, Department of Mathematics, University of British Columbia, VanCOUVER, BC V6T 1Z2, CANAda

E-mail address: dghioca@math.ubc.ca

Liang-Chung Hsia, Department of Mathematics, National Taiwan Normal University, TAIWAN, ROC

E-mail address: hsia@math.ntnu.edu.tw 